\title{
A NEW PROOF THAT METRIC SPACES ARE PARACOMPACT
}

\author{
MARY ELLEN RUDIN
}

By using a well-ordered open cover, there is a simple proof of the nice theorem [1] that every metric space is paracompact.

Assume that $X$ is a metric space and that $\left\{C_{\alpha}\right\}$ is an open cover of $X$ indexed by ordinals. Let $\rho$ be a metric on $X$ and let $S(x, r)$ be the open sphere with center $x$ and radius $r$. For each positive integer $n$ define $D_{\alpha n}$ (by induction on $n$ ) to be the union of all spheres $S\left(x, 2^{-n}\right)$ such that:

(1) $\alpha$ is the smallest ordinal with $x \in C_{\alpha}$,

(2) $x \in D_{\beta j}$ if $j<n$,

(3) $S\left(x, 3 \cdot 2^{-n}\right) \subset C_{\alpha}$.

Then $\left\{D_{\alpha n}\right\}$ is a locally finite refinement of $\left\{C_{\alpha}\right\}$ which covers $X$; hence $X$ is paracompact.

Certainly $\left\{D_{\alpha n}\right\}$ refines $\left\{C_{\alpha}\right\}$. To see that $\left\{D_{\alpha n}\right\}$ covers $X$, observe that, for $x \in X$, there is a smallest ordinal $\alpha$ such that $x \in C_{\alpha}$, and an $n$ so large that (3) holds. Then, by (2), $x \in D_{\beta j}$ for some $j \leqq n$.

To prove that $\left\{D_{\alpha n}\right\}$ is locally finite, assume an $x \in X$ and let $\alpha$ be the smallest ordinal such that $x \in D_{\alpha n}$ for some $n$, and choose $j$ so that $S\left(x, 2^{-j}\right) \subset D_{\alpha n}$. The proof consists of showing that:

(a) if $i \geqq n+j, S\left(x, 2^{-n-j}\right)$ intersects no $D_{\beta i}$,

(b) if $i<n+j, S\left(x, 2^{-n-j}\right)$ intersects $D_{\beta i}$ for at most one $\beta$.

Proof of (a). Since $i>n$, by (2), every one of the spheres of radius $2^{-i}$ used in the definition of $D_{\beta i}$ has its center $y$ outside of $D_{\alpha n}$. And since $S\left(x, 2^{-j}\right) \subset D_{\alpha n}, \rho(x, y) \geqq 2^{-j}$. But $i \geqq j+1$ and $n+j \geqq j+1$, so $S\left(x, 2^{-n-j}\right) \cap S\left(y, 2^{-i}\right)=\varnothing$.

Proof of (b). Suppose $p \in D_{\beta i}, q \in D_{\gamma i}$, and $\beta<\gamma$; we want to show that $\rho(p, q)>2^{-n-j+1}$. There are points $y$ and $z$ such that $p \in S\left(y, 2^{-i}\right)$ $\subset D_{\beta i}, q \in S\left(z, 2^{-i}\right) \subset D_{\gamma_{i}}$; and, by (3), $S\left(y, 3 \cdot 2^{-i}\right) \subset C_{\beta}$ but, by (2), $z \notin C_{\beta}$. So $\rho(y, z) \geqq 3 \cdot 2^{-i}$ and $\rho(p, q)>2^{-i} \geqq 2^{-n-j+1}$.

\section{BIBLIOGRAPHY}

1. A. H. Stone, Paracompactness and product spaces, Bull. Amer. Math. Soc. 54 (1948), 977-982.

\section{UNIVERSITY OF WISCONSIN}

Received by the editors January 30, 1968. 